\title{
Parametric Analysis of Entropy Generation in Off-centered Stagnation Flow Towards a Rotating Disc
}

\begin{abstract}
The similarity solution for the steady stagnation flow towards an off-centered rotating disc is gives a system of non-linear partial differential equations. These nonlinear differential equations are numerically solved by applying well known Keller-Box Method. After finding the velocity distributions, the important designing subject, entropy generation of this system has been analyzed. Graphical results are presented to investigate effects of the rotation ratio $\alpha$, off-centering, Reynolds number and axial height on the radial and azimuthal velocities and entropy generation. In order to show the effectiveness of the KellerBox method, the obtained results are compared with available solutions obtained using DTM. The obtained results demonstrate the reliability of the algorithm and the KellerBox method is an attractive method in solving the systems of nonlinear partial differential equations, and also the entropy generation is an important parameter depends on design and work conditions that should be in the attention of designers of these rotating systems.
\end{abstract}

Keywords: Keller-Box method, entropy generation, Offcentering, Reynolds number

M.M. Rashidi: University of Michigan-Shanghai Jiao Tong University Joint Institute, Shanghai Jiao Tong University, Shanghai, Peoples Republic of China, E-mail:mm_rashidi@sjtu.edu.ca, mm_rashidi@yahoo.com

L. Shamekhi: Mechanical Engineering Department, Engineering Faculty of Bu-Ali Sina University, Hamedan, Iran

*Corresponding Author: Sunil Kumar: Department of Mathematics, National Institute of Technology, Jamshedpur, 801014, Jharkhand, India, E-mail: skumar.math@nitjsr.ac.in, skiitbhu28@gmail.com

\section{Nomenclature}

a

$b$

$p$

$r$

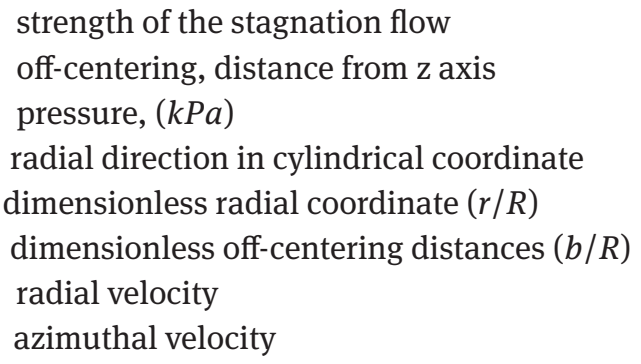

$w \quad$ axial velocity

$x$ horizontal direction in Cartesian coordinate

$y$ vertical direction in Cartesian coordinate

$z$ axial direction in cylindrical coordinate

\section{Greek letters}

$\beta$ stretching factor of mesh generation in axial direction

$\Omega$ angular velocity of rotating disk

$\theta$ azimuthal direction in cylindrical coordinate

$\mu$ fluid viscosity

$v$ kinematic viscosity

$\rho$ fluid density

\section{Introduction}

Most phenomena in real world are described through nonlinear equations which some of them are solved using numerical methods. Because of the nonlinearities in the reduced differential equation, no analytical solution is available and the nonlinear equation is usually solved numerically subject to boundary conditions, one of which is prescribed at infinity.

The Keller-Box method reported by Keller has become popular for obtaining non similar solutions for boundary these problems [1]. The basic idea of the Keller-Box method is to write the governing system of equations in the form of a first-order system [2]. The method has been particularly useful in tackling that class of problem, which although non similar, may be formulated as a progression between known limiting similarity states which are inherent in the physical configuration under examination.

The Keller-Box scheme [3, 4, 5] for the solution of equations is both accurate and robust. As a consequence it has been used extensively in solving a broad class of problems including convection flows [6], jet flows [7, 8], turbulent boundary layers $[5,9]$ as well as separating flows $[10]$.

The problem of flow over a single rotating disk is one of the classical problems in the fluid mechanics that 
has received much attention in several industrial and engineering processes i.e. rotating machinery, lubrication, oceanography and computer storage devices. Some direct applications are wastewater treatment, electrochemical engineering, turbo-machinery, centrifugal pumps, sport disks, rotating blades, flight mechanics and chemical vapor deposition processes [11]. Von Karman [12] originally studied hydrodynamic flow due to an infinite rotating disk. In his work, Von Karman introduced his famous appropriate transformations, giving rise to ordinary differential equations which are a reduced form of the governing partial differential equations. He introduced the similarity transformations which reduced the governing partial differential equations to ordinary differential equations.

Afterwards, different physical aspects of the von Karman flow over rotating disk were vastly explored by numerous authors [13, 14, 15]. Cochran [13] obtained a more accurate solution of von Karman's problem using the numerical integration of momentum equation.

Stuart [14], Sparrow and Gregg [15] and others extended the problem by taking suction and blowing effects at the disk surface and discussed the flow pattern by applying various quantitative assumptions on suction and injection parameters. Sparrow and Gregg considered the removal of the condensate using centrifugal forces on a cooled rotating disk. Following von Karman's study of a rotating disk in an infinite fluid, Sparrow and Gregg transformed the Navier-Stokes equations into a set of nonlinear ordinary differential equations and numerically integrated them for the similarity solution for several finite film thicknesses.

Hiemenz [16] was the first one who studied two dimensional stagnation point flow over a flat plate. The three dimensional flow towards a stretching flat sheet was investigated by Wang [17] for the axisymmetric case.

In recent years, many studies have been published on the rotating disk with different methods and for different side parameters, for example the desirable disk is porous medium, or the analysis has been done in the presence of magneto-hydrodynamic flow [18]. Some of these studies are done by analytic methods. Rashidi et al. [19, 20, 21, $22,23]$ analyzed rotating disk with different conditions by different analytic methods like DTM, HAM and etc.

In recent years, many studies have been published on the applications and entropy generation rates of the second law of thermodynamics. Aiboud and Saouli [24] illustrated the application of the second law analysis of thermodynamics to viscoelastic magneto-hydrodynamic flow over a stretching surface analytically using Kummer's functions. The effect of viscous dissipation and thermal radiation on entropy generation in Blasius flow was dis- played numerically by Butt et al. [25]. The results showed that as the thermal radiation parameter increases, the produced entropy decreases. San et al. [26] depicted the entropy generation analysis for the combined forced convection heat and mass transfer in a two dimensional channel.

Entropy generation analysis, which is a thermodynamic approach, is used to optimize the thermal engineering devices for higher energy efficiency [27]. The performance of engineering equipment in the presence of the irreversibilities is reduced and entropy generation is a measure of the level of the available irreversibilities in a process. Entropy generation can be used as a quantitative measure of irreversibilities that is associated with a process, because of this fact that; the greater the entropy generation indicates the greater the extent of irreversibilities. It is important to note that the second law of thermodynamics is more reliable than the first law of thermodynamics analysis, because of the limitation of the first law efficiency in a heat transfer engineering systems and also heat transfer, mass transfer, viscous dissipation, etc. can be used as the sources of entropy generation.

Here, we have considered the off-centered stagnation flow towards a rotating disc. The main goal of the present study is to apply Keller-Box scheme to the problem and analyze the entropy generation in this case.

Therefore, this paper has been organized as follows. In Section 2, the flow analysis and mathematical formulation are presented. In Section 3 basic idea of Keller-Box method and its implementation on the governing equations have been demonstrated. In Section 4, entropy generation has been extended. Section 6 contains the results and discussion. The conclusions are summarized in Section 7.

\section{Flow analysis and mathematical formulation}

We consider the off-centered stagnation flow towards a rotating disc [28]. Fig. 1 shows a stagnation flow along the $z$ axis impinging on a rotating disc whose axis is distance $b$ from that axis. The Navier-Stokes equations for cylindrical coordinate system $(r, \theta, z)$ are as follows:

$$
\begin{gathered}
\frac{1}{r} \frac{\partial}{\partial r}(r u)+\frac{1}{r} \frac{\partial v}{\partial \theta}+\frac{\partial w}{\partial z}=0 \\
\rho\left[\frac{\partial u}{\partial t}+u \frac{\partial u}{\partial r}+\frac{v}{r} \frac{\partial u}{\partial \theta}+w \frac{\partial u}{\partial z}-\frac{v^{2}}{r}\right]+\frac{\partial p}{\partial r} \\
=\mu\left[\frac{\partial^{2} u}{\partial r^{2}}+\frac{1}{r} \frac{\partial u}{\partial r}+\frac{1}{r^{2}} \frac{\partial^{2} u}{\partial \theta^{2}}+\frac{\partial^{2} u}{\partial z^{2}}-\frac{u}{r^{2}}-\frac{2}{r^{2}} \frac{\partial v}{\partial \theta}\right]+\rho g_{r}
\end{gathered}
$$




$$
\begin{aligned}
& \rho\left[\frac{\partial v}{\partial t}+u \frac{\partial v}{\partial r}+\frac{v}{r} \frac{\partial v}{\partial \theta}+w \frac{\partial v}{\partial z}+\frac{u v}{r}\right]+\frac{1}{r} \frac{\partial p}{\partial \theta} \\
& =\mu\left[\frac{\partial^{2} v}{\partial r^{2}}+\frac{1}{r} \frac{\partial v}{\partial r}+\frac{1}{r^{2}} \frac{\partial^{2} v}{\partial \theta^{2}}+\frac{\partial^{2} v}{\partial z^{2}}-\frac{v}{r^{2}}+\frac{2}{r^{2}} \frac{\partial u}{\partial \theta}\right]+\rho g_{\theta},
\end{aligned}
$$

$$
\begin{aligned}
& \rho\left[\frac{\partial w}{\partial t}+u \frac{\partial w}{\partial r}+\frac{v}{r} \frac{\partial w}{\partial \theta}+w \frac{\partial w}{\partial z}\right]+\frac{\partial p}{\partial z} \\
& =\mu\left[\frac{\partial^{2} w}{\partial r^{2}}+\frac{1}{r} \frac{\partial w}{\partial r}+\frac{1}{r^{2}} \frac{\partial^{2} w}{\partial \theta^{2}}+\frac{\partial^{2} w}{\partial z^{2}}\right]+\rho g_{z},
\end{aligned}
$$

The conditions at infinity correspond to the potential stagnation flow:

$$
u=a x, \quad v=a y, \quad w=-2 a z .
$$

Some thought reveals the conditions on the disc are:

$$
u=-\Omega y, \quad v=-\Omega(x-b), \quad w=0 .
$$

The following similarity transforms are then introduced:

$$
\begin{gathered}
u=a x f^{\prime}(\eta)-\Omega y g(\eta)+b \Omega k(\eta), \\
v=a y f^{\prime}(\eta)-\Omega x g(\eta)+b \Omega h(\eta), \\
w=-2 \sqrt{a v} f(\eta),
\end{gathered}
$$

and:

$$
\eta=\sqrt{a / v} z
$$

The induced flows $h$ and $k$ are necessary. After some work, the constant property Navier-Stokes equations reduce to

$$
\left(f^{\prime}\right)^{2}-\alpha^{2} g^{2}-2 f^{\prime} f^{\prime \prime}=1+f^{\prime \prime \prime}
$$

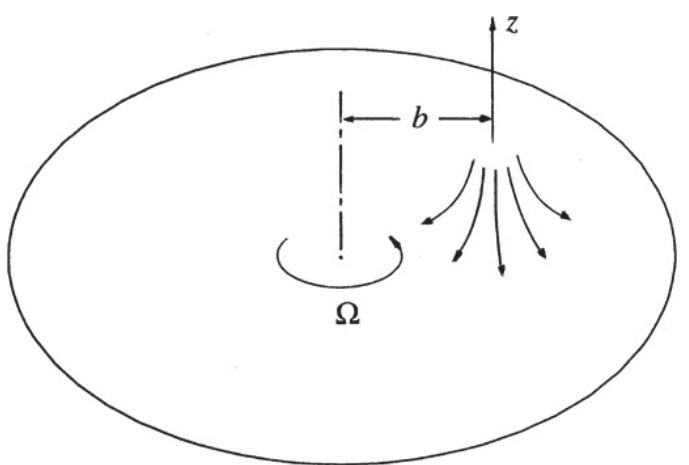

Fig. 1. Off-centered stagnation flow on a rotating disc.

$$
\begin{gathered}
2 g f^{\prime}-2 f g^{\prime}=g^{\prime \prime}, \\
k f^{\prime}-\alpha h g-2 f k^{\prime}=k^{\prime \prime}, \\
\alpha k g+h f^{\prime}-2 f h^{\prime}=h^{\prime \prime},
\end{gathered}
$$

where $\alpha=\Omega / a$ is an important non-dimensional parameter. The boundary conditions become

$$
(0)=f^{\prime}(0)=0, \quad f^{\prime}(\infty)=1 \text {, }
$$

$$
\begin{array}{ll}
g(0)=1, & g(\infty)=0, \\
k(0)=0, & k(\infty)=0, \\
h(0)=1, & h(\infty)=0 .
\end{array}
$$

Given $\alpha$, we first solve for the functions $f$ and $g$ from Eqs. (11), (12), (15) and (16) then solve for the functions $k$ and $h$ from Eqs. (13), (14), (17) and (18).

\section{Implementation of Keller-Box method}

For non-linear differential equation, one way to obtain the solution is to linearize the differential equation before it is written in finite-difference form. Starting from a first approximation, higher order iterates can be obtained by solving linearized equation.

Consider the third-order non-linear differential Eq. (11) and the second-order non-linear differential Eqs. (12), (13) and (14) with the boundary conditions Eqs. (15), (16), (17) and (18).

Before the implementation of finite difference method, first, Eqs. (11) to (14) should be replaced by a system of first order ordinary differential equation. $f, f^{\prime}, f^{\prime \prime}$ are denoted by $f, a, b$ respectively, so the system of three first order equations instead of Eq. (11) can be written as:

$$
\begin{aligned}
& f^{\prime}=a, \\
& f^{\prime \prime}=b, \\
& f^{\prime \prime \prime}=a^{2}-\alpha^{2} g^{2}-2 f b-1 .
\end{aligned}
$$

Again $g, g^{\prime}$ are denoted by $g, c$ respectively and the system of two first order equations instead of Eq. (12) will be:

$$
\begin{aligned}
& g^{\prime}=c, \\
& g^{\prime \prime}=2 g a-2 f c .
\end{aligned}
$$


Also $k, k^{\prime}$ are denoted by $k, d$ and $h, h^{\prime}$ by $h, e$ respectively and the systems of two first order equations instead of Eqs. (13) and (14) can be written as:

$$
\begin{aligned}
& k^{\prime}=d, \\
& k^{\prime \prime}=k a-\alpha h g-2 f d, \\
& h^{\prime}=e, \\
& h^{\prime \prime}=\alpha k g+h a-2 f e .
\end{aligned}
$$

With employment the quantities midway between net points, Centering about the midpoint and employing Newton's linearization method, differences approximation of Eqs. (19) to (23) can be respectively written as:

$$
\begin{aligned}
& \delta f_{i}-\delta f_{i-1}-d i s_{i}\left(\delta a_{i-1 / 2}\right)=X_{1, i}, \\
& \delta a_{i}-\delta a_{i-1}-d i s_{i}\left(\delta b_{i-1 / 2}\right)=X_{2, i}, \\
& \delta b_{i}-\delta b_{i-1}+\frac{d i s_{i}}{2}\left(-2 a_{i} \delta a_{i}-2 a_{i-1} \delta a_{i-1}+2 \alpha^{2} g_{i} \delta g_{i}\right. \\
& \left.+2 \alpha^{2} g_{i-1} \delta g_{i-1}+2 f_{i} \delta b_{i}+2 f_{i-1} \delta b_{i-1}+2 b_{i} \delta f_{i}+2 b_{i-1} \delta f_{i-1}\right) \\
& =X_{3, i},
\end{aligned}
$$

and:

$$
\begin{aligned}
& \delta g_{i}-\delta g_{i-1}-d i s_{i}\left(\delta c_{i-1 / 2}\right)=X_{4, i} \\
& \delta c_{i}-\delta c_{i-1}+\frac{d i s_{i}}{2}\left(-2 g_{i} \delta a_{i}-2 g_{i-1} \delta a_{i-1}-2 a_{i} \delta g_{i}\right. \\
& \left.-2 a_{i-1} \delta g_{i-1}+2 f_{i} \delta c_{i}+2 f_{i-1} \delta c_{i-1}+2 c_{i} \delta f_{i}+2 c_{i-1} \delta f_{i-1}\right) \\
& =X_{5, i},
\end{aligned}
$$

also:

$$
\begin{aligned}
& \delta k_{i}-\delta k_{i-1}-d i s_{i}\left(\delta d_{i-1 / 2}\right)=X_{6, i} \\
& d_{i}-\delta d_{i-1}+\frac{d i s_{i}}{2}\left(-k_{i} \delta a_{i}-k_{i-1} \delta a_{i-1}-a_{i} \delta k_{i}-a_{i-1} \delta k_{i-1}\right. \\
& +\alpha h_{i} \delta g_{i}+\alpha h_{i-1} \delta g_{i-1}+\alpha g_{i} \delta h_{i}+\alpha g_{i-1} \delta h_{i-1} \\
& \left.+2 f_{i} \delta d_{i}+2 f_{i-1} \delta d_{i-1}+2 d_{i} \delta f_{i}+2 d_{i-1} \delta f_{i-1}\right)=X_{7, i},
\end{aligned}
$$

and:

$$
\begin{aligned}
& \delta h_{i}-\delta h_{i-1}-d i s_{i}\left(\delta e_{i-1 / 2}\right)=X_{8, i} \\
& \delta e_{i}-\delta e_{i-1}+\frac{d i s_{i}}{2}\left(-\alpha k_{i} \delta g_{i}-\alpha k_{i-1} \delta g_{i-1}-\alpha g_{i} \delta k_{i}\right. \\
& -\alpha g_{i-1} \delta k_{i-1}-h_{i} \delta a_{i}-h_{i-1} \delta a_{i-1}-a_{i} \delta h_{i}-a_{i-1} \delta h_{i-1} \\
& \left.+2 f_{i} \delta e_{i}+2 f_{i-1} \delta e_{i-1}+2 e_{i} \delta f_{i}+2 e_{i-1} \delta f_{i-1}\right)=X_{9, i}
\end{aligned}
$$

where:

$$
\begin{aligned}
& X_{1, i}=f_{i-1}-f_{i}+\operatorname{dis}_{i}\left(a_{i-1 / 2}\right), \\
& X_{2, i}=a_{i-1}-a_{i}+\operatorname{dis}_{i}\left(b_{i-1 / 2}\right), \\
& X_{3, i}=b_{i-1}-b_{i}+\operatorname{dis}_{i}\left(a_{i-1 / 2}^{2}-\alpha^{2} g_{i-1 / 2}^{2}-2 f_{i-1 / 2} b_{i-1 / 2}-1\right),
\end{aligned}
$$

and:

$$
\begin{aligned}
& X_{4, i}=g_{i-1}-g_{i}+\operatorname{dis}_{i}\left(c_{i-1 / 2}\right), \\
& X_{5, i}=c_{i-1}-c_{i}+\operatorname{dis}_{i}\left(2 g_{i-1 / 2} a_{i-1 / 2}-2 f_{i-1 / 2} c_{i-1 / 2}\right),
\end{aligned}
$$

and also:

$$
\begin{aligned}
X_{6, i}= & k_{i-1}-k_{i}+d i s_{i}\left(d_{i-1 / 2}\right), \\
X_{7, i}= & d_{i-1}-d_{i}+d i s_{i}\left(k_{i-1 / 2} a_{i-1 / 2}-\alpha h_{i-1 / 2} g_{i-1 / 2}\right. \\
& \left.-2 f_{i-1 / 2} d_{i-1 / 2}\right), \\
X_{8, i}= & h_{i-1}-h_{i}+d i s_{i}\left(e_{i-1 / 2}\right), \\
X_{9, i}= & e_{i-1}-e_{i}+d i s_{i}\left(\alpha k_{i-1 / 2} g_{i-1 / 2}+h_{i-1 / 2} a_{i-1 / 2}\right. \\
& \left.-2 f_{i-1 / 2} e_{i-1 / 2}\right) .
\end{aligned}
$$

Where $i$ index is contour and $d i s_{i}$ is non-uniform step size in $\eta$ direction. This spatial step is obtained by:

$$
\delta \eta=\frac{\eta(\beta-1)}{\beta^{n+1}-1}, \quad \operatorname{dis}_{i}=\delta \eta \times \beta^{i-1} .
$$

where $n$ is number of points in calculation domain in $\eta$ direction.

To complete the system the boundary conditions will be recalled:

$$
\begin{gathered}
\delta f_{1}=0, \quad \delta a_{1}=0, \quad \delta g_{1}=0, \quad \delta k_{1}=0, \quad \delta h_{1}=0, \\
\delta a_{M}=0, \quad \delta g_{M}=0, \quad \delta k_{M}=0, \quad \delta h_{M}=0 .
\end{gathered}
$$

The Eqs. (23) to (32) have a block tridiagonal structure. A block tridiagonal matrix is a block matrix, which is having square matrices (blocks) in the lower, main, and upper diagonal, where all other blocks is a zero matrices. It is basically a tridiagonal matrix but has submatrices in places of scalars. This is not obvious, and to simplify the solution, the system would be written in matrix vector form. After that the matrix equation can be presented as below:

$$
[A][\delta]=[r] .
$$

That can be expanded more as:

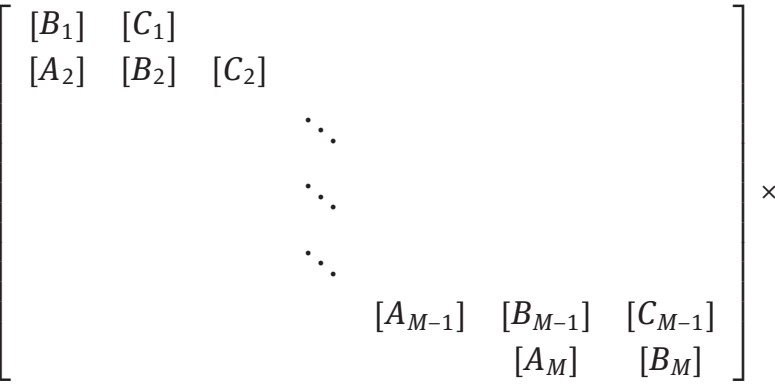

$$
\begin{aligned}
& {\left[\begin{array}{c}
{\left[\delta_{1}\right]} \\
{\left[\delta_{2}\right]} \\
\vdots \\
{\left[\delta_{M-1}\right]} \\
{\left[\delta_{M}\right]}
\end{array}\right]=\left[\begin{array}{c}
{\left[r_{1}\right]} \\
{\left[r_{2}\right]} \\
\vdots \\
{\left[r_{M-1}\right]} \\
{\left[r_{M}\right]}
\end{array}\right],}
\end{aligned}
$$


where $\left[A_{i}\right],\left[B_{i}\right],\left[C_{i}\right]$ are $9 \times 9$ matrixes and $\left[\delta_{i}\right],\left[r_{i}\right]$ are $9 \times 1$ matrixes as:

$$
\delta_{i}=\left[\begin{array}{c}
\delta f_{i} \\
\delta a_{i} \\
\delta g_{i} \\
\delta k_{i} \\
\delta h_{i} \\
\delta b_{i} \\
\delta c_{i} \\
\delta d_{i} \\
\delta e_{i}
\end{array}\right], r_{i}=\left[\begin{array}{c}
X_{5, i} \\
X_{6, i} \\
X_{7, i} \\
X_{8, i} \\
X_{9, i} \\
X_{1, i+1} \\
X_{2, i+1} \\
X_{3, i+1} \\
X_{4, i+1}
\end{array}\right], r_{1}=\left[\begin{array}{c}
0 \\
0 \\
0 \\
0 \\
0 \\
X_{1,2} \\
X_{2,2} \\
X_{3,2} \\
X_{4,2}
\end{array}\right], r_{M}=\left[\begin{array}{c}
X_{5, M} \\
X_{6, M} \\
X_{7, M} \\
X_{8, M} \\
X_{9, M} \\
0 \\
0 \\
0 \\
0
\end{array}\right]
$$

Note that the first five rows of $\left[r_{1}\right]$ and the last four rows of $\left[r_{M}\right]$ are zero because of boundary conditions have been presented in (32).

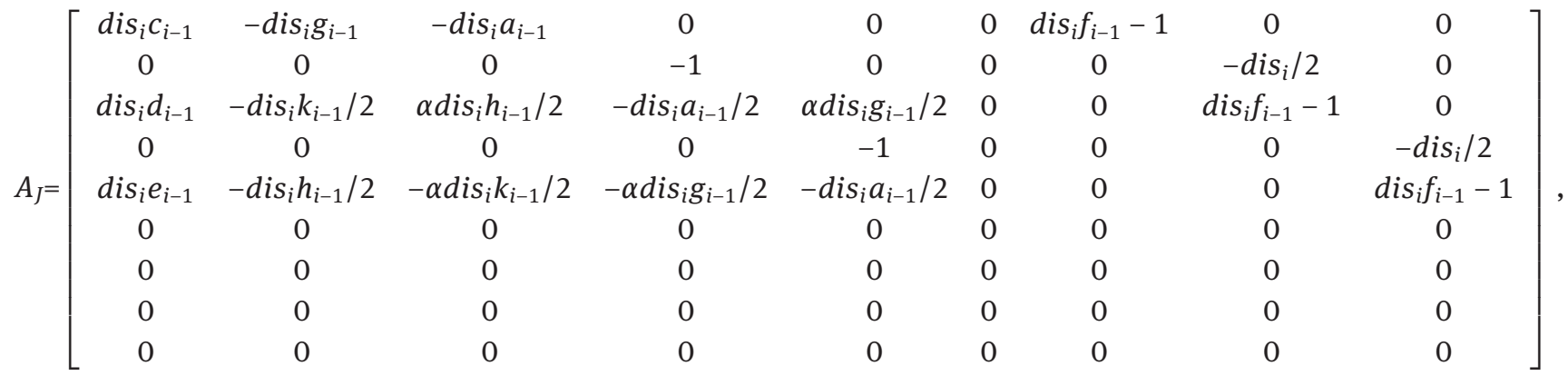

and:

$B_{J}=\left[\begin{array}{ccccccccc}d i s_{i} c_{i} & -d i s_{i} g_{i} & -d i s_{i} a_{i} & 0 & 0 & 0 & d i s_{i} f_{i}+1 & 0 & 0 \\ 0 & 0 & 0 & 1 & 0 & 0 & 0 & -d i s_{i} / 2 & 0 \\ d i s_{i} d_{i} & -d i s_{i} k_{i} / 2 & \alpha d i s_{i} h_{i} / 2 & -d i s_{i} a_{i} / 2 & \alpha d i s_{i} g_{i} / 2 & 0 & 0 & d i s_{i} f_{i}+1 & 0 \\ 0 & 0 & 0 & 0 & 1 & 0 & 0 & 0 & -d i s_{i} / 2 \\ d i s_{i} e_{i} & -d i s_{i} h_{i} / 2 & -\alpha d i s_{i} k_{i} / 2 & -\alpha d i s_{i} g_{i} / 2 & -d i s_{i} a_{i} / 2 & 0 & 0 & 0 & d i s_{i} f_{i}+1 \\ -1 & -d i s_{i+1} / 2 & 0 & 0 & 0 & 0 & 0 & 0 & 0 \\ 0 & -1 & 0 & 0 & 0 & -d i s_{i+1} / 2 & 0 & 0 & 0 \\ d i s_{i+1} b_{i} & -d i s_{i+1} a_{i} & \alpha^{2} d i s_{i+1} g_{i} & 0 & 0 & d i s_{i+1} f_{i}-1 & 0 & 0 & 0 \\ 0 & 0 & -1 & 0 & 0 & 0 & -d i s_{i+1} / 2 & 0 & 0\end{array}\right]$,

and also:

$$
C_{J}=\left[\begin{array}{ccccccccc}
0 & 0 & 0 & 0 & 0 & 0 & 0 & 0 & 0 \\
0 & 0 & 0 & 0 & 0 & 0 & 0 & 0 & 0 \\
0 & 0 & 0 & 0 & 0 & 0 & 0 & 0 & 0 \\
0 & 0 & 0 & 0 & 0 & 0 & 0 & 0 & 0 \\
0 & 0 & 0 & 0 & 0 & 0 & 0 & 0 & 0 \\
1 & -d i s_{i+1} / 2 & 0 & 0 & 0 & 0 & 0 & 0 & 0 \\
0 & 1 & 0 & 0 & 0 & -d i s_{i+1} / 2 & 0 & 0 & 0 \\
d i s_{i+1} b_{i+1} & -d i s_{i+1} a_{i+1} & \alpha^{2} d i s_{i+1} g_{i+1} & 0 & 0 & d i s_{i+1} f_{i+1}+1 & 0 & 0 & 0 \\
0 & 0 & 1 & 0 & 0 & 0 & -d i s_{i+1} / 2 & 0 & 0
\end{array}\right],
$$


In this matrix formulation, there should be attention to first and last row of matrixes. Here $C_{1}$ matrix can obtained by $C_{i}$ and $A_{M}$ also by $A_{j}$ with no changes. About others:

$$
B_{1}=\left[\begin{array}{ccccccccc}
1 & 0 & 0 & 0 & 0 & 0 & 0 & 0 & 0 \\
0 & 1 & 0 & 0 & 0 & 0 & 0 & 0 & 0 \\
0 & 0 & 1 & 0 & 0 & 0 & 0 & 0 & 0 \\
0 & 0 & 0 & 1 & 0 & 0 & 0 & 0 & 0 \\
0 & 0 & 0 & 0 & 1 & 0 & 0 & 0 & 0 \\
-1 & -d i s_{2} / 2 & 0 & 0 & 0 & 0 & 0 & 0 & 0 \\
0 & -1 & 0 & 0 & 0 & -d i s_{2} / 2 & 0 & 0 & 0 \\
d i s_{2} b_{1} & -d i s_{2} a_{1} & \alpha^{2} d i s_{2} g_{1} & 0 & 0 & \text { dis }_{2} f_{1}-1 & 0 & 0 & 0 \\
0 & 0 & -1 & 0 & 0 & 0 & -d i s_{2} / 2 & 0 & 0
\end{array}\right],
$$

and:

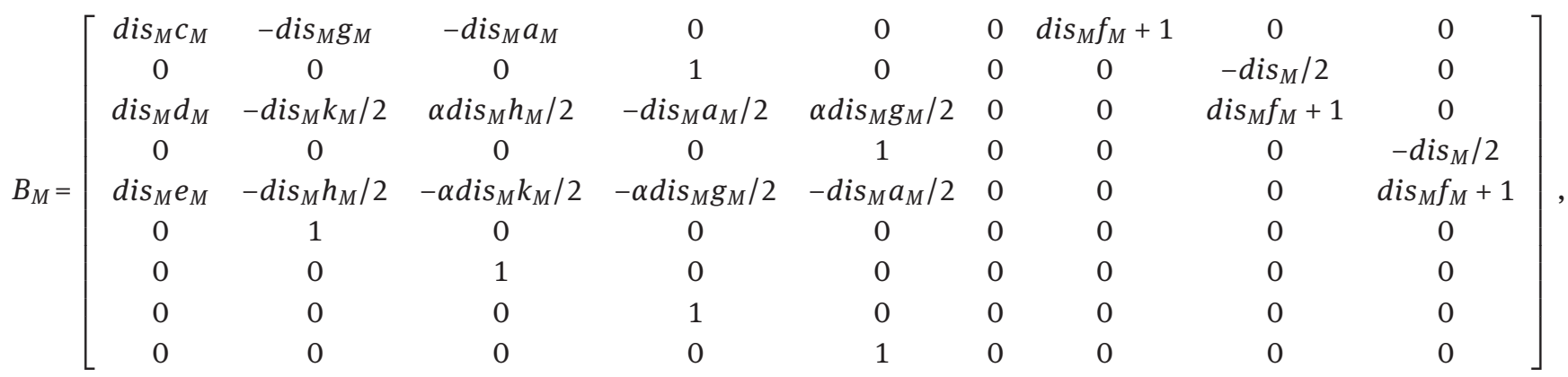

and in addition:

$$
A_{1}=0, \quad C_{M}=0
$$

Note that the first five rows of $\left[B_{1}\right]$ and the last four rows of $\left[B_{M}\right]$ are in specific form to satisfy boundary conditions in coupling with Eq. (35).

As seen the resulting finite difference Eqs. (23) to (30) were nonlinear algebraic equations. The differential equations in finite difference forms were written first and then the resulting nonlinear algebraic equations were linearized by Newton's method. Then a block-tridiagonal factorization scheme is applied on the coefficient matrix of the finite-difference equations. Now this block-tridiagonal matrix can be solved easily.

\section{Entropy generation}

It has been shown [29] the general entropy generation rate for non-reacting flows and a non-radiating fluid when the only external body forces are due to gravity and the only non-uniformities leading to chemical potentials are due to temperature and composition differences. Some studies [30, 31] have simplified this expression. However this simplification masks some important physical aspects. Generally, the local volumetric entropy generation rate [32, 33] in this problem is:

$$
\dot{S}_{\text {gen }}^{\prime \prime \prime}=\frac{\mu}{T_{w}}\left(2\left[\left(\frac{\partial u}{\partial r}\right)^{2}+\frac{1}{r^{2}}\left(\frac{\partial v}{\partial \theta}+u\right)^{2}+\left(\frac{\partial w}{\partial z}\right)^{2}\right]+\left(\frac{\partial v}{\partial z}+\frac{1}{r} \frac{\partial w}{\partial \theta}\right)^{2}+\left(\frac{\partial w}{\partial r}+\frac{\partial u}{\partial z}\right)^{2}+\left[\frac{1}{r} \frac{\partial u}{\partial \theta}+r \frac{\partial}{\partial r}\left(\frac{v}{r}\right)\right]^{2}\right) \cdot
$$

The above equation reveals that the entropy generation is due to fluid friction irreversibility effect. By neglecting less effective components of this equation comparing effective ones, it means neglecting the radial and azimuthal partial differences; this equation will be written as:

$$
\dot{S}_{g e n}^{\prime \prime \prime}=\frac{\mu}{T_{w}}\left(2\left[\frac{1}{r^{2}}(u)^{2}+\left(\frac{\partial w}{\partial z}\right)^{2}\right]+\left(\frac{\partial v}{\partial z}\right)^{2}+\left(\frac{\partial u}{\partial z}\right)^{2}\right) .
$$


By applying Eqs. (7) to (10), every parts of this equation can be written as:

$$
\begin{aligned}
\frac{2}{r^{2}}(u)^{2}= & \frac{2}{r^{2}}\left(a^{2} x^{2} f^{\prime 2}+\Omega^{2} y^{2} g^{2}+\Omega^{2} b^{2} k^{2}+2 a b x \Omega k f^{\prime}-2 a x y \Omega g f^{\prime}-2 b y \Omega^{2} g k\right), \\
2\left(\frac{\partial w}{\partial z}\right)^{2}=2 & \left(-2 \sqrt{a v} \cdot f^{\prime} \cdot \sqrt{a / v}\right)^{2}=2\left(-2 a f^{\prime}\right)^{2}=8 a^{2} f^{\prime 2}, \\
\left(\frac{\partial v}{\partial z}\right)^{2}= & \left(a y f^{\prime \prime} \cdot \sqrt{a / v}-\Omega x g^{\prime} \cdot \sqrt{a / v}+\Omega b h^{\prime} \cdot \sqrt{a / v}\right)^{2}=\left(a^{3} y^{2} / v\right) f^{\prime \prime 2} \\
& +\left(a x^{2} \Omega^{2} / v\right) g^{\prime 2}+\left(a b^{2} \Omega^{2} / v\right) h^{\prime 2}+\left(2 a^{2} b y \Omega / v\right) f^{\prime \prime} h^{\prime}-\left(2 a^{2} x y \Omega / v\right) f^{\prime \prime} g^{\prime}-\left(2 a b x \Omega^{2} / v\right) g^{\prime} h^{\prime}, \\
\left(\frac{\partial u}{\partial z}\right)^{2}= & \left(a x f^{\prime \prime} \cdot \sqrt{a / v}-\Omega y g^{\prime} \cdot \sqrt{a / v}+\Omega b k^{\prime} \cdot \sqrt{a / v}\right)^{2}=\left(a^{3} x^{2} / v\right) f^{\prime \prime 2} \\
& +\left(a y^{2} \Omega^{2} / v\right) g^{\prime 2}+\left(a b^{2} \Omega^{2} / v\right) k^{\prime 2}+\left(2 a^{2} b x \Omega / v\right) f^{\prime \prime} k^{\prime}-\left(2 a^{2} x y \Omega / v\right) f^{\prime \prime} g^{\prime}-\left(2 a b y \Omega^{2} / v\right) g^{\prime} k^{\prime} .
\end{aligned}
$$

Now by factoring the variables and using Eq. (44), it's clear that Eq. (43) can be written in this pattern:

$$
\begin{aligned}
\dot{S}_{g e n}^{\prime \prime \prime}= & \frac{\mu}{T_{w}}\left(c_{1} f^{\prime \prime 2}+c_{2} f^{\prime 2}+c_{3} g^{\prime 2}+c_{4} h^{\prime 2}+c_{5} k^{\prime 2}+c_{6} g^{2}-c_{7} k^{2}+c_{8} k f^{\prime}+c_{9} g f^{\prime}+c_{10} g k+c_{11} f^{\prime \prime} h^{\prime}+c_{12} f^{\prime \prime} k^{\prime}\right. \\
& \left.+c_{13} f^{\prime \prime} g^{\prime}+c_{14} g^{\prime} h^{\prime}+c_{15} g^{\prime} k^{\prime}\right),
\end{aligned}
$$

where $c_{i}, \quad i=1,15$ are coefficients that by defining $R e=\frac{\Omega R^{2}}{v}$ as the rotational Reynolds number will be obtained as below:

$$
\begin{aligned}
f^{\prime \prime 2}\left(a^{3} y^{2} / v+a^{3} x^{2} / v\right) & =f^{\prime \prime}\left(a^{3} r^{2} / v\right)=f^{\prime \prime 2}\left(\frac{1}{\alpha} a^{2}\left(\frac{r}{R}\right)^{2} R e\right), \\
f^{\prime 2}\left(\frac{2}{r^{2}} a^{2} x^{2}+8 a^{2}\right) & =f^{\prime 2}\left(2 a^{2} \cos ^{2} \theta+8 a^{2}\right), \\
g^{\prime 2}\left(a x^{2} \Omega^{2} / v+a y^{2} \Omega^{2} / v\right) & =g^{\prime 2}\left(a r^{2} \Omega^{2} / v\right)=g^{\prime 2}\left(\alpha a^{2}\left(\frac{r}{R}\right)^{2} R e\right), \\
h^{\prime 2}\left(a b^{2} \Omega^{2} / v\right) & =h^{\prime 2}\left(\alpha a^{2}\left(\frac{b}{R}\right)^{2} R e\right), \\
k^{\prime 2}\left(a b^{2} \Omega^{2} / v\right) & =k^{\prime 2}\left(\alpha a^{2}\left(\frac{b}{R}\right)^{2} R e\right), \\
g^{2}\left(\frac{2}{r^{2}} \Omega^{2} y^{2}\right) & =g^{2}\left(2 \Omega^{2} \sin ^{2} \theta\right)=g^{2}\left(2 a^{2} \alpha^{2} \sin ^{2} \theta\right), \\
k^{2}\left(\frac{2}{r^{2}} \Omega^{2} b^{2}\right) & =k^{2}\left(2 a^{2} \alpha^{2}\left(\frac{b}{R}\right)^{2}\right), \\
k f^{\prime}\left(\frac{2}{r^{2}} 2 a b x \Omega\right) & =k f^{\prime}\left(4 a^{2} \alpha \cos \theta \frac{b}{r}\right)=k f^{\prime}\left(4 a^{2} \alpha \cos \theta\left(\frac{b}{R} / \frac{r}{R}\right)\right), \\
g f^{\prime}\left(-\frac{2}{r^{2}} 2 a x y \Omega\right) & =g f^{\prime}\left(-4 a^{2} \alpha \sin \theta \cos \theta\right), \\
g k\left(-\frac{2}{r^{2}} 2 b y \Omega^{2}\right) & =g k\left(-4 a^{2} \alpha^{2} \sin \theta \frac{b}{r}\right)=g k\left(-4 a^{2} \alpha^{2} \sin \theta\left(\frac{b}{R} / \frac{r}{R}\right)\right), \\
f^{\prime \prime} h^{\prime}\left(2 a^{2} b y \Omega / v\right) & =f^{\prime \prime} h^{\prime}\left(2 a^{2} b y \Omega / v\right)=f^{\prime \prime} h^{\prime}\left(2 a^{2} \operatorname{Resin} \theta\left(\frac{b}{R} \times \frac{r}{R}\right)\right), \\
f^{\prime \prime} k^{\prime}\left(2 a^{2} b x \Omega / v\right) & =f^{\prime \prime} k^{\prime}\left(2 a^{2} \operatorname{Re} \cos \theta\left(\frac{b}{R} \times \frac{r}{R}\right)\right), \\
f^{\prime \prime} g^{\prime}\left(-2 a^{2} x y \Omega / v\right) & =f^{\prime \prime} g^{\prime}\left(-4 a^{2} \operatorname{Re} \cos \theta \sin \theta\left(\frac{r}{R}\right)^{2}\right), \\
g^{\prime} h^{\prime}\left(-2 a b x \Omega^{2} / v\right) & =g^{\prime} h^{\prime}\left(-2 a^{2} \alpha \operatorname{Re} \cos \theta\left(\frac{b}{R} \times \frac{r}{R}\right)\right), \\
k^{\prime}\left(-2 a b \Omega^{2} / v\right) & =g^{\prime} k^{\prime}\left(-2 a^{2} \alpha R e \sin \theta\left(\frac{b}{R} \times \frac{r}{R}\right)\right) .
\end{aligned}
$$


The entropy generation number, dimensionless form of entropy generation rate, represents the ratio between the actual entropy generation rate and the characteristic entropy generation rate. It is more useful to drive and analyze dimensionless form of entropy generation rate.

The similarity transformation parameters of Eqs. (7) to (10) are employed to non-dimensionalize the local entropy generation given in Eq. (45), thus the entropy generation number becomes:

$$
\begin{aligned}
\dot{S}_{g e n}^{\prime \prime \prime}= & \frac{\mu}{T_{w}}\left(f^{\prime 2}\left[8+2 \cos ^{2} \theta\right]+g^{\prime 2}\left[\alpha \bar{r}^{2} \times R e\right]\right. \\
& +h^{\prime 2}\left[\alpha \overline{\bar{r}}^{2} \times R e\right]+f^{\prime \prime 2}\left[\frac{1}{\alpha} \bar{r}^{2} \times R e\right]+k^{2}\left[\alpha \overline{\bar{r}}^{2} \times R e\right] \\
& +g^{2}\left[2 \alpha^{2} \sin ^{2} \theta\right]+k^{2}\left[2 \alpha^{2} \overline{\bar{r}}^{2}\right]+f^{\prime} k[4 \alpha \cos \theta \times \overline{\bar{r}} / \bar{r}] \\
& +f^{\prime} g[-4 \alpha \cos \theta \times \sin \theta]+g k\left[-4 \alpha^{2} \sin \theta \times \overline{\bar{r}} / \bar{r}\right] \\
& +f^{\prime \prime} h^{\prime}[2 \sin \theta \times \operatorname{Re} \times \overline{\bar{r}} \times \bar{r}]+f^{\prime \prime} k^{\prime}[2 \cos \theta \times \operatorname{Re} \times \overline{\bar{r}} \times \bar{r}] \\
& +f^{\prime \prime} g^{\prime}\left[-4 \bar{r}^{2} \cos \theta \times \sin \theta \times R e\right] \\
& +g^{\prime} h^{\prime}[-2 \alpha \cos \theta \times \operatorname{Re} \times \overline{\bar{r}} \times \bar{r}] \\
& \left.+g^{\prime} k^{\prime}[-2 \alpha \sin \theta \times \operatorname{Re} \times \overline{\bar{r}} \times \bar{r}]\right),
\end{aligned}
$$

where $\bar{r}=\frac{r}{R}, \overline{\bar{r}}=\frac{b}{R}$ are dimensionless defined parameters.

\section{Results and discussion}

The nonlinear differential Eqs. (11) to (14) subject to the boundary conditions (15) to (18) have been solved numerically via Keller-Box method for value of $\alpha$. Then the entropy generation of this problem has been analyzed for some values of off-centering and Reynolds number. Graphical representation of results is very useful to demonstrate the efficiency and accuracy of the numerical Keller-Box method for above problem. In order to verify the accuracy of our present method, we have compared our results with those of analytical method using DTM. Fig. 2 shows $f^{\prime}(\eta)$, $g(\eta)$ and the induced velocity functions $h(\eta)$ and $k(\eta)$ due to the non-alignment of the stagnation flow and the rotating disc obtained by the Keller-Box and the DTM with for the case of rotation ratio $\alpha=7$. It concluded that, the results obtained by the DTM, only valid for small values of independent variable $\eta$ and these series diverge around infinity. So, the solutions are obtained by the Keller-Box scheme are accurate and efficient by changing rotation ratio and in all range of $\eta$.

Fig. 3 shows the function $f^{\prime}(\eta)$, for various rotation ratios $\alpha$. For $\alpha=0$ or Homann's stagnation flow [34], $f^{\prime}(\eta)$ increases monotonically to the finite velocity caused by the pressure gradient at infinity. When $\alpha$ is greater than

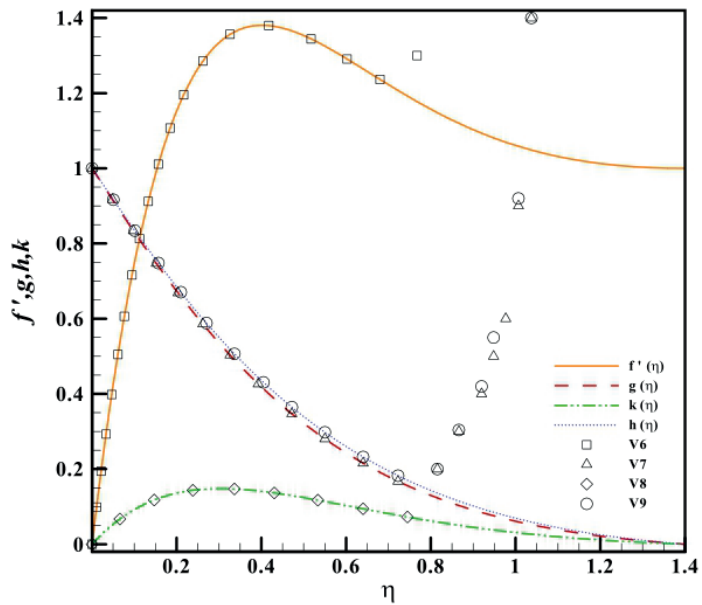

Fig. 2. Numerical solution of $f^{\prime}(\eta), g(\eta), h(\eta), k(\eta)$ obtained by Keller-Box method and the analytical solution of the DTM [35] for $\alpha=7$.

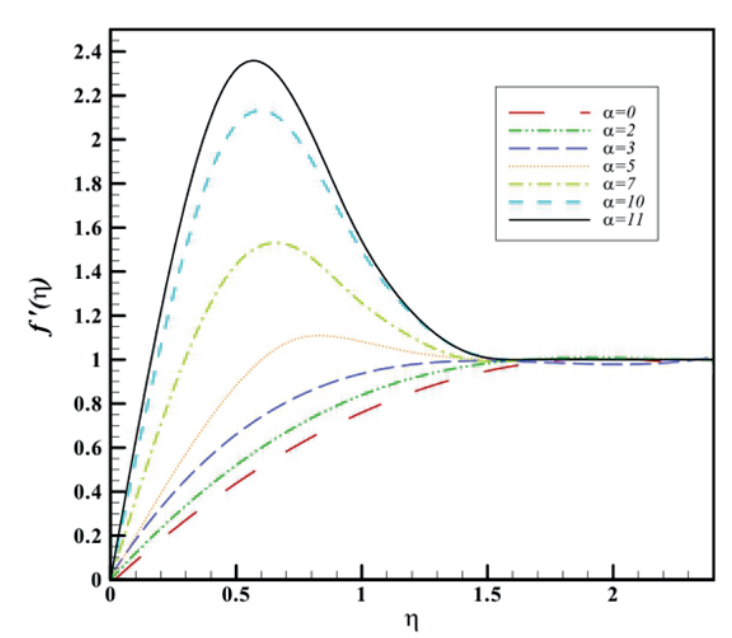

Fig. 3. $f^{\prime}(\eta)$ Obtained by the Keller-Box method for $\alpha=0,2,3,5,7$, $10,11$.

3.4 there is an overshoot of velocity. This overshoot, that is progressively larger for larger $\alpha$, is due to the centrifugal forces of the rotating disc. Because $\alpha$ is quadratic form in Eq. (11) and because $\alpha$ is multiplied by nonlinear terms, therefore by increasing $\alpha$, Eqs. (11) - (14) become more nonlinear that this causes diverge of numerical and analytical solutions. As can be seen all curves in this figure approach unity within a boundary layer of about 2.5. Fig. 4 shows $g(\eta)$ and Figs. 5 and 6 show the induced velocity functions $h(\eta)$ and $k(\eta)$ due to the non-alignment of the stagnation flow and the rotating disc. According to Fig. 4 it can be seen that for all values of $\alpha$, the procedures of approaching to zero are the same, the only effect of $\alpha$ is the speed of this approach. As can be seen, when $\alpha$ increases, $g(\eta)$ approaches to zero in smaller distance of $\eta$. 


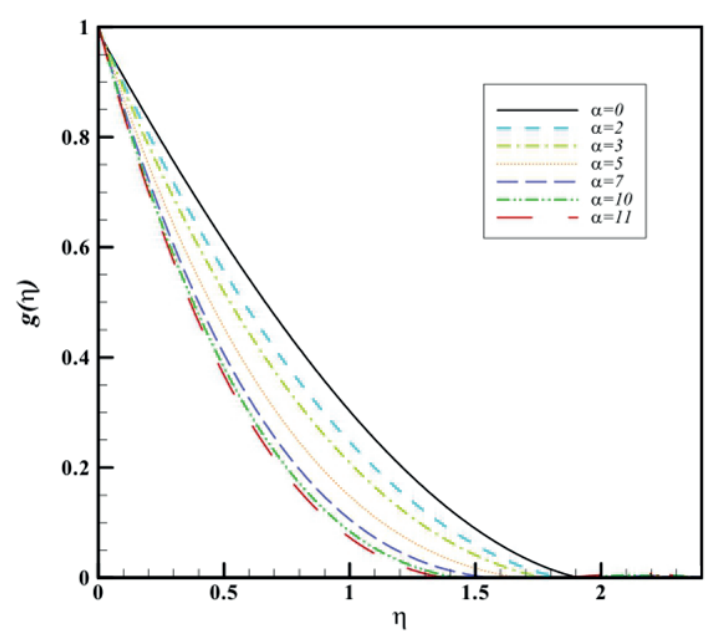

Fig. 4. $g(\eta)$ Obtained by the Keller-Box method for $\alpha=0,2,3,5,7$, $10,11$.

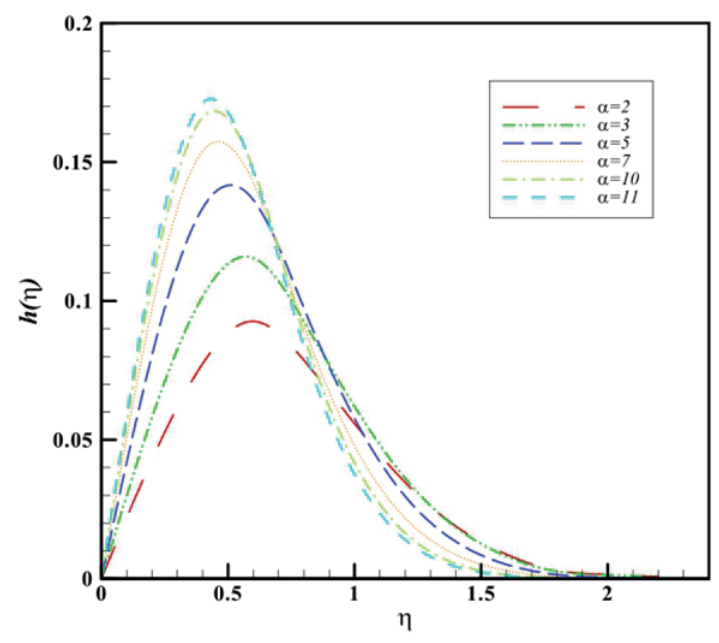

Fig. 5. $h(\eta)$ Obtained by the Keller-Box method for $\alpha=0,2,3,5,7$, $10,11$.

Fig. 7 illustrates the effect of no dimensional offcentering on velocity distribution in the radial direction, $u(\eta)$ and Figs. 8 and 9 illustrate the effect of Reynolds number on velocity distribution in the radial direction, $u(\eta)$ and azimuthal direction, $v(\eta)$ of stagnation flow toward a rotating disk respectively. In these Figs. the constant parameters are height of $\mathrm{Z}=0.1$ and $\alpha=7$.

According to Eqs. (7) and (8), in very small $\eta$, here $\mathrm{Z}=0.1$, that only $g(\eta)$ and $k(\eta)$ are nonzero, it is clear that change of Reynolds number only appears as a coefficient multiple in both radial and azimuthal velocities whit no change in the shape of velocity's contours. Also it can be seen that in these small $\eta$, no dimensional off-centering has no effect on azimuthal velocity whereas it's effective on radial velocity. The results presented in Figs. 7 to 9 show

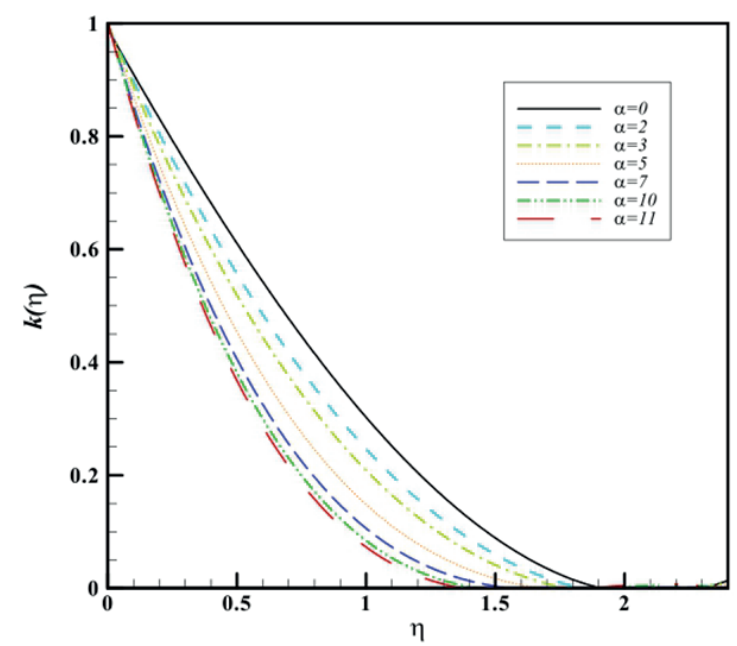

Fig. 6. $k(\eta)$ Obtained by the Keller-Box method for $\alpha=0,2,3,5,7$, $10,11$.

these predictions as good as possible, so there is a very good agreement between the results and physics of problem.

Figs. 10 and 11 present the effect of no dimensional off-centering on velocity distribution in the radial direction, $u(\eta)$ and azimuthal direction, $v(\eta)$. Here the height parameter is $Z=0.5$. In this height of $\eta$, all of functions exist. Figs. 12 and 13 show the effect of Reynolds number on these two velocity distributions. Again, as it can be obtained, the Reynolds number's effect appears as a coefficient multiple in both radial and azimuthal velocities whit no change in the shape of velocity's contours.

In the infinity height of boundary layer, about $Z=1.4$, since the only remained function is $f^{\prime}(\eta)$, so based on Eqs. (7) and (8), no dimensional off-centering has no effect on velocity distributions and Reynolds number again only have coefficient effect. So there is no presentation on these in this height.

The results of no dimensional entropy generation for different conditions of off-centering, Reynolds number and height of solutions are presented in Figs. 14 to 19. By increasing in no dimensional off-centering, the no dimensional entropy generation increases in all heights of $\eta$. It can be seen that this effect is smaller at infinity than other heights. Also it can be seen that when height approaches to infinity, the entropy generation decreases. Another subject in these Figs. is about Reynolds number effect; by increasing the Reynolds number entropy generation increases and its position approach to bigger radial boundaries. This effect can be seen in all heights, much more in smaller heights. 


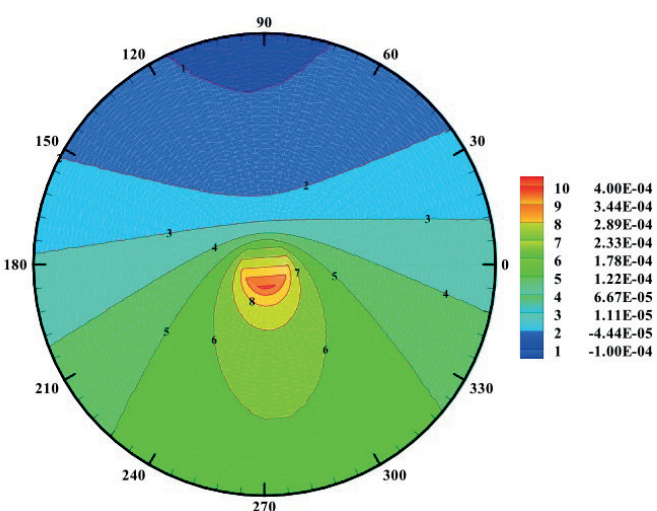

(a)

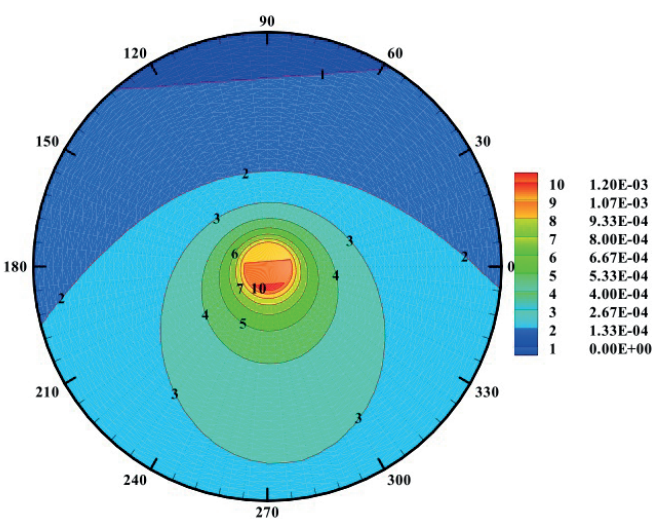

(b)

Fig. 7. Effect of no dimensional off-centering ( $\mathrm{b} / \mathrm{R}=0.2 ; \mathrm{b} \mathrm{b} / \mathrm{R}=0.8$ ) on velocity distribution in the radial $(r)$ direction, $u(\eta)$, of stagnation flow toward a rotating disk with $R e=100$, in height of $\mathrm{Z}=0.1$ obtained by the Keller-Box method $\alpha=7$.

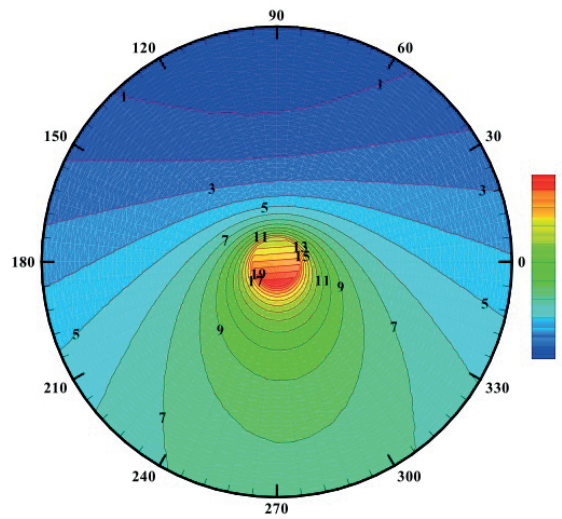

(a)

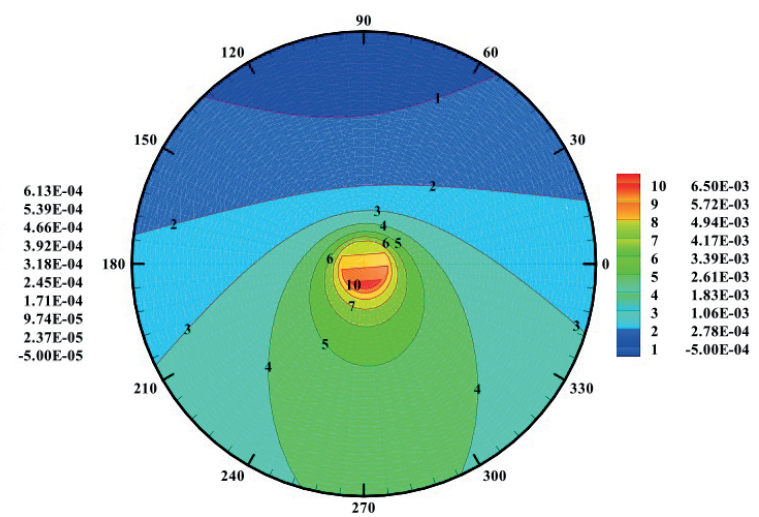

(b)

Fig. 8. Effect of Reynolds number (a $R e=10$; $\mathrm{b} R e=100$ ) on velocity distribution in the radial $(r)$ direction, $u(\eta)$, of stagnation flow toward a rotating disk, for no dimensional off-centering $b / R=0.4$, in height of $Z=0.1$ obtained by the Keller-Box method $\alpha=7$.

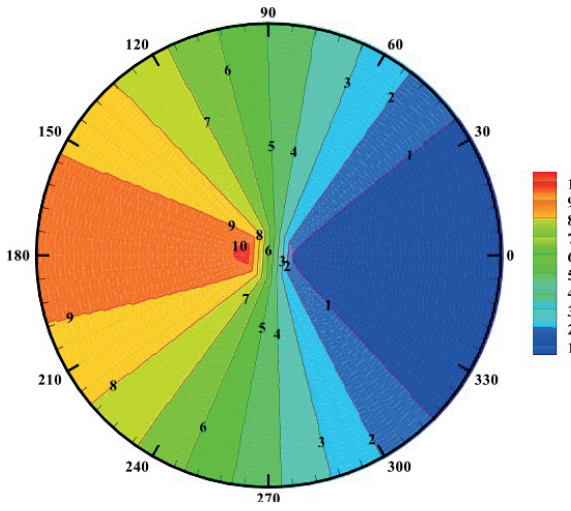

(a)

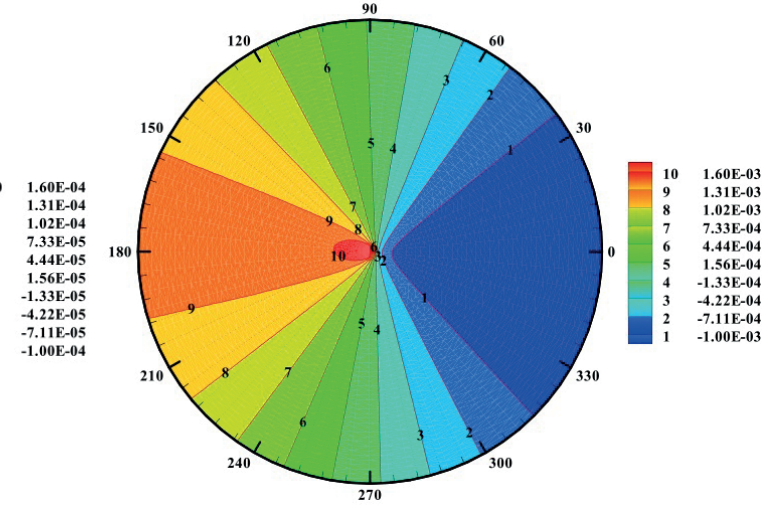

(b)

Fig. 9. Effect of Reynolds number ( $\operatorname{Re}=10$; $\mathrm{b} R e=100$ ) on velocity distribution in the azimuthal $(\theta)$ direction, $v(\eta)$, of stagnation flow toward a rotating disk, for no dimensional off-centering $\mathrm{b} / \mathrm{R}=0.4$, in height of $\mathrm{Z}=0.1$ obtained by the Keller-Box method $\alpha=7$. 


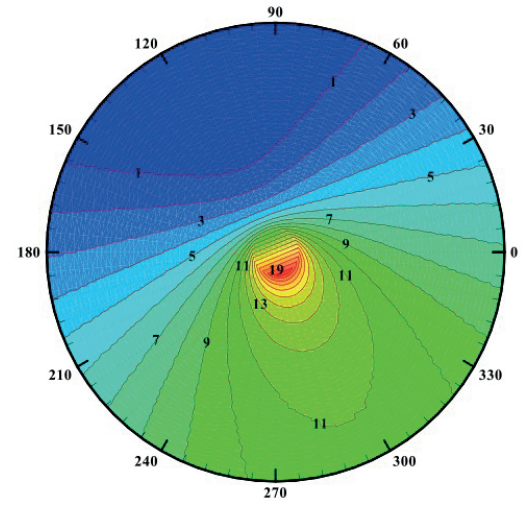

(a)

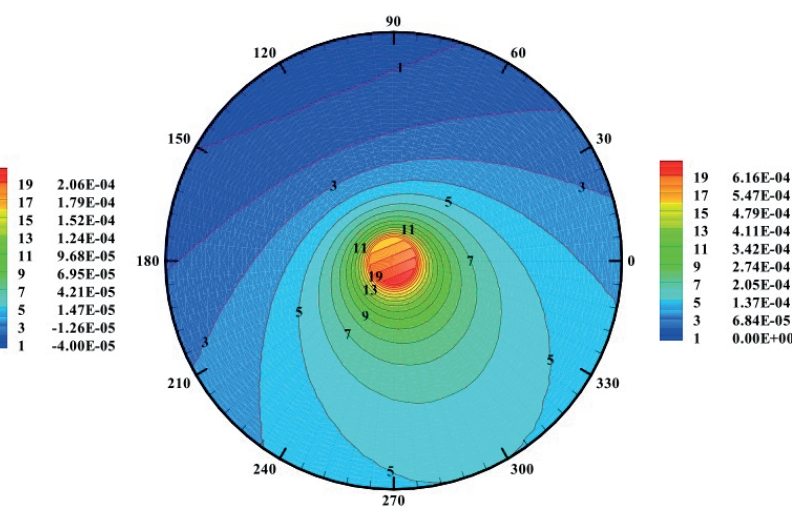

(b)

Fig. 10. Effect of no dimensional off-centering $(\mathrm{a} b / \mathrm{R}=0.2 ; \mathrm{b} b / \mathrm{R}=0.8)$ on velocity distribution in the radial $(r)$ direction, $u(\eta)$, of stagnation flow toward a rotating disk with $R e=100$, in height of $Z=0.5$ obtained by the Keller-Box method $\alpha=7$.

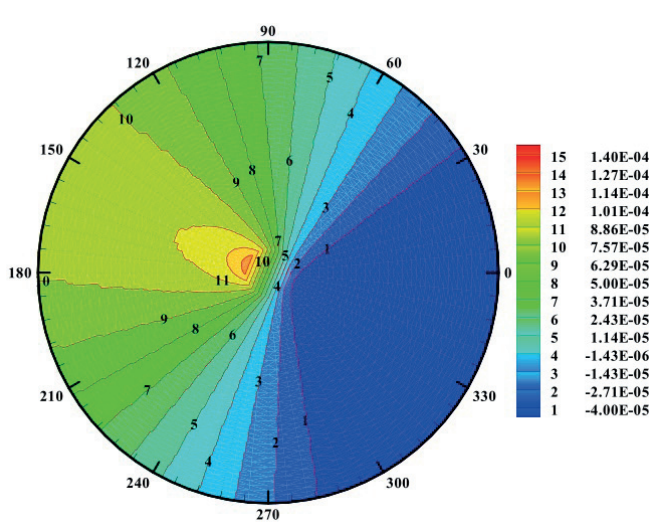

(a)

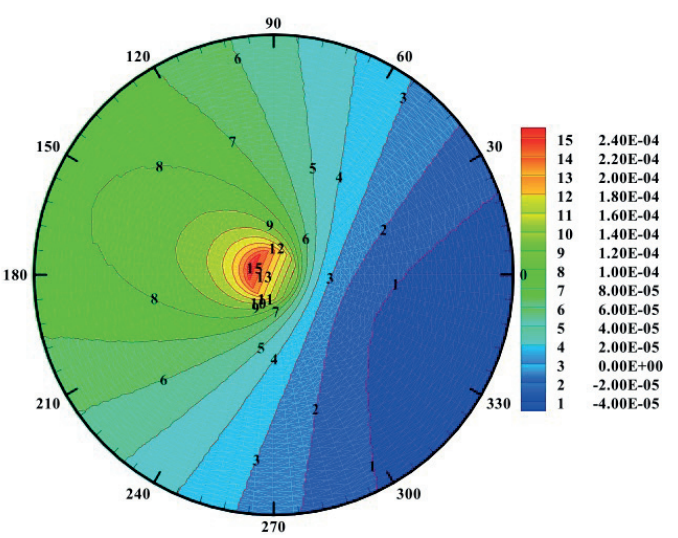

(b)

Fig. 11. Effect of no dimensional off-centering $(a b / R=0.2 ; b b / R=0.8)$ on velocity distribution in the azimuthal $(\theta)$ direction, $v(\eta)$, of stagnation flow toward a rotating disk with $R e=100$, in height of $Z=0.5$ obtained by the Keller-Box method $\alpha=7$.

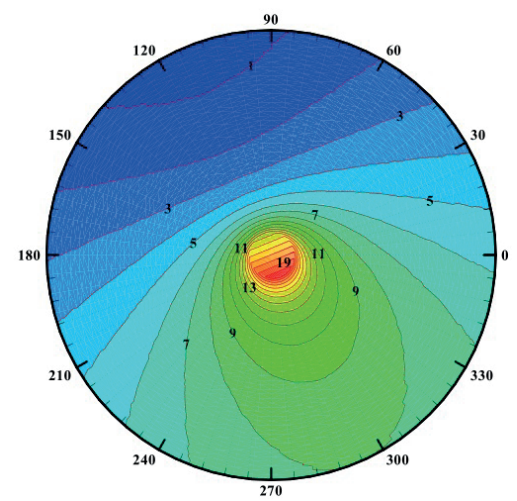

(a)

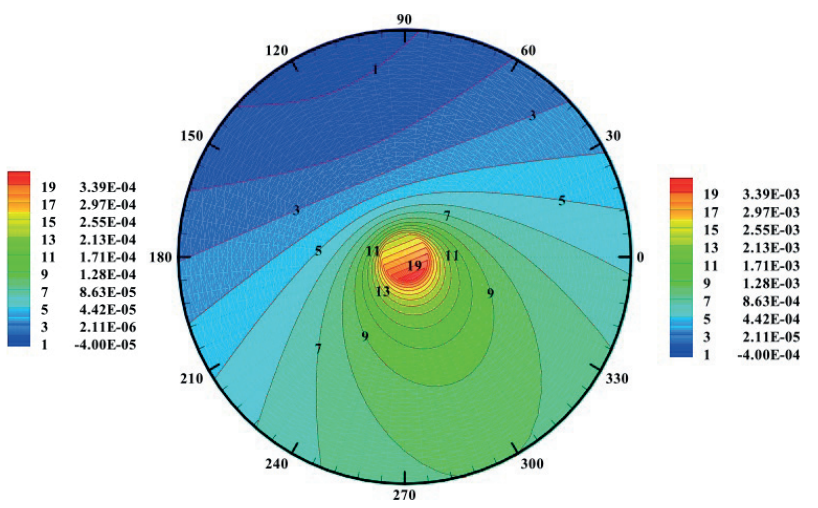

(b)

Fig. 12. Effect of Reynolds number (a $R e=10$; $\mathrm{b} R e=100$ ) on velocity distribution in the radial ( $r$ ) direction, $u(\eta)$, of stagnation flow toward a rotating disk, for no dimensional off-centering $\mathrm{b} / \mathrm{R}=0.4$, in height of $\mathrm{Z}=0.5$ obtained by the Keller-Box method $\alpha=7$. 


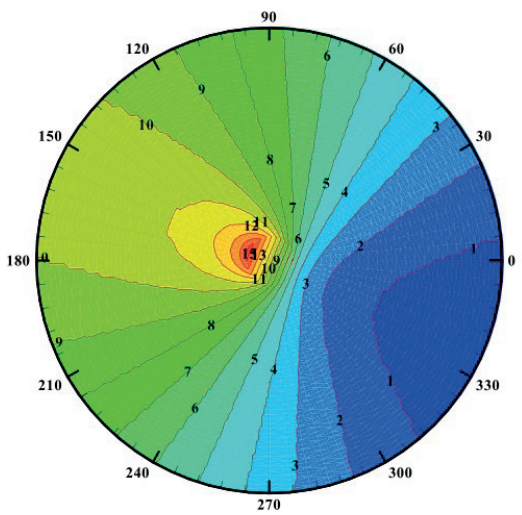

(a)

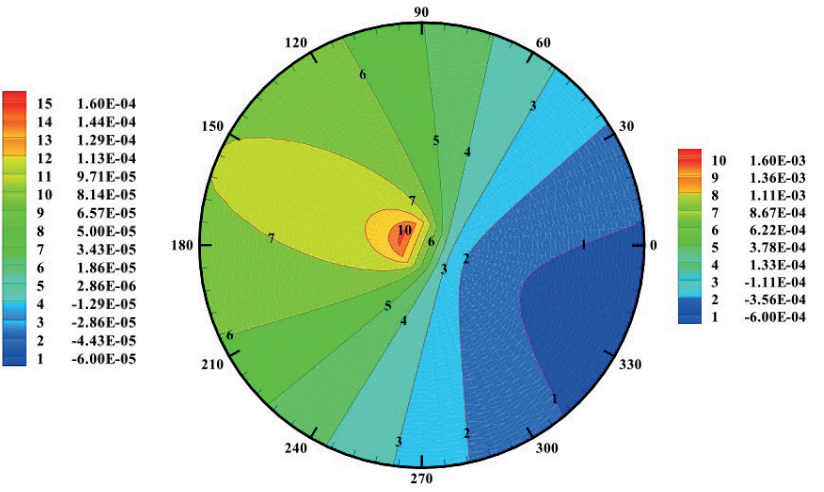

(b)

Fig. 13. Effect of Reynolds number ( $\mathrm{a} e=10 ; \mathrm{b} R e=100$ ) on velocity distribution in the azimuthal $(\theta)$ direction, $v(\eta)$, of stagnation flow toward a rotating disk, for no dimensional off-centering $b / R=0.4$, in height of $Z=0.5$ obtained by the Keller-Box method $\alpha=7$.

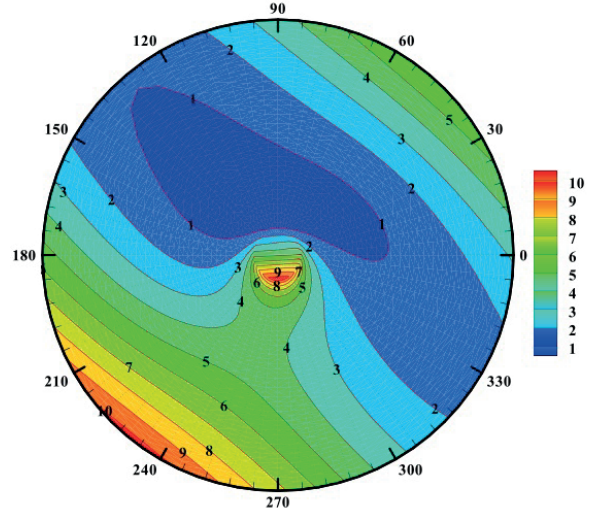

(a)

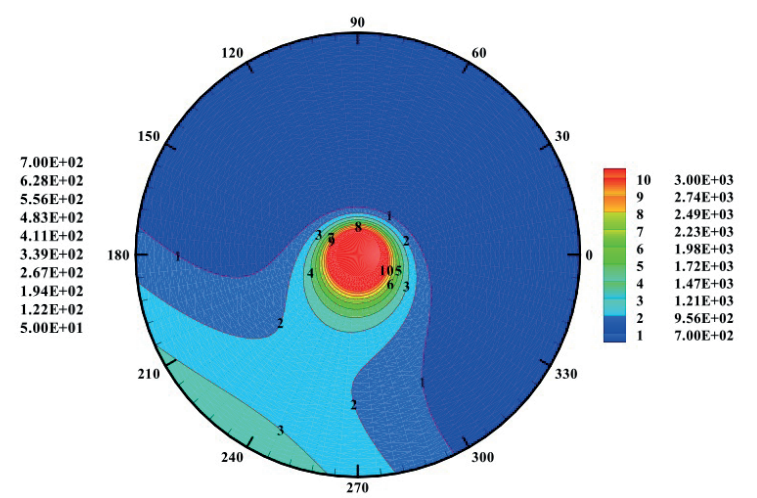

(b)

Fig. 14. Effect of no dimensional off-centering $(a b / R=0.2 ; b b / R=0.8)$ on dimensionless entropy generation of stagnation flow toward $a$ rotating disk with $R e=100$, in height of $Z=0.1$ obtained by the Keller-Box method $\alpha=7$.

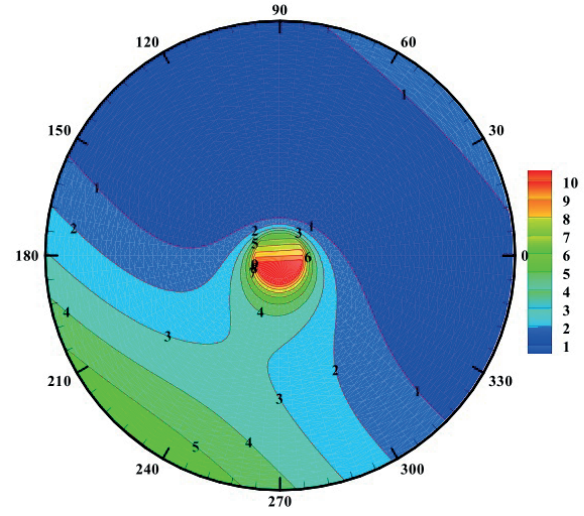

(a)

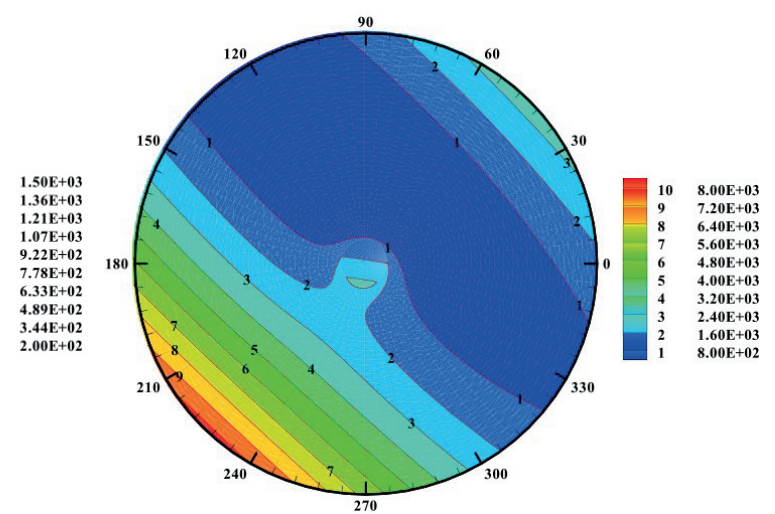

(b)

Fig. 15. Effect of Reynolds number ( $\mathrm{R} e=10$; $\mathrm{b} R e=100$ ) on dimensionless entropy generation of stagnation flow toward a rotating disk, for no dimensional off-centering $b / R=0.4$, in height of $Z=0.1$ obtained by the Keller-Box method $\alpha=7$. 


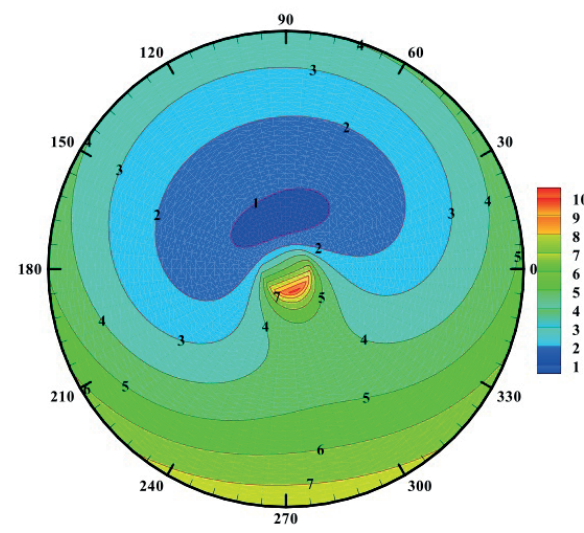

(a)

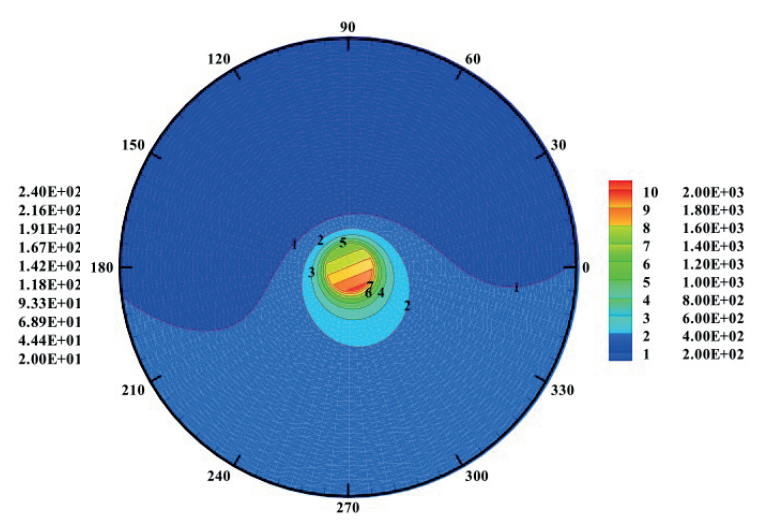

(b)

Fig. 16. Effect of no dimensional off-centering $(a b / R=0.2 ; b b / R=0.8)$ on dimensionless entropy generation of stagnation flow toward a rotating disk with $R e=100$, in height of $Z=0.1$ obtained by the Keller-Box method $\alpha=7$.

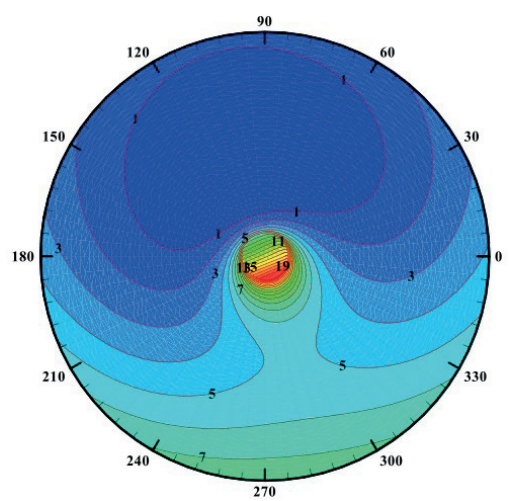

(a)

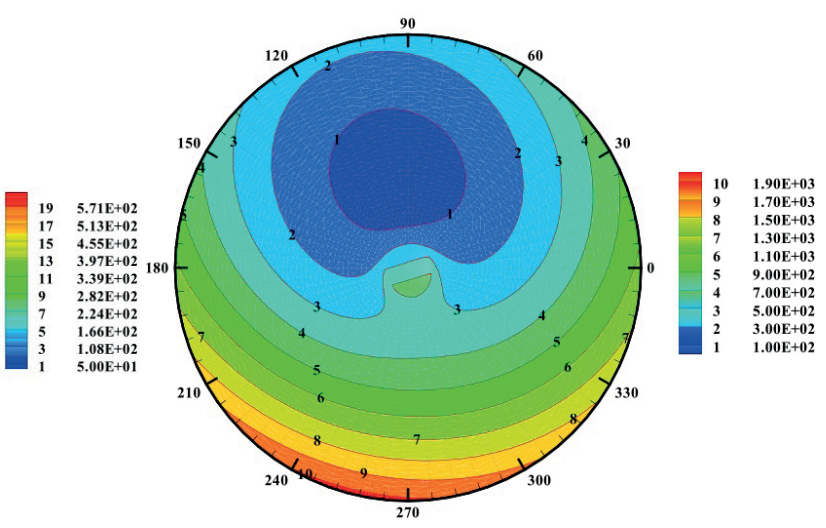

(b)

Fig. 17. Effect of Reynolds number (a $R e=10$; $\mathrm{b} R e=100$ ) on dimensionless entropy generation of stagnation flow toward a rotating disk, for no dimensional off-centering $b / R=0.4$, in height of $Z=0.5$ obtained by the Keller-Box method $\alpha=7$.

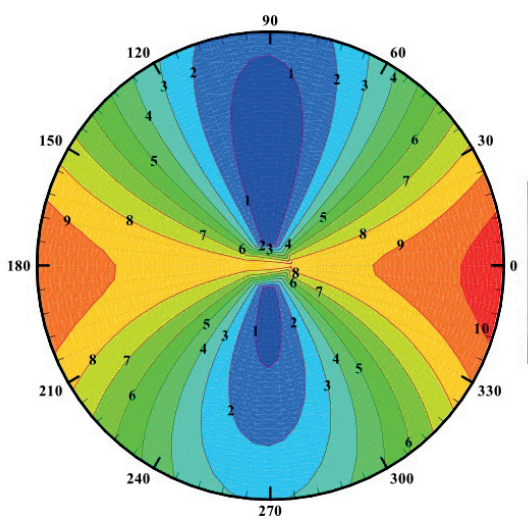

(a)

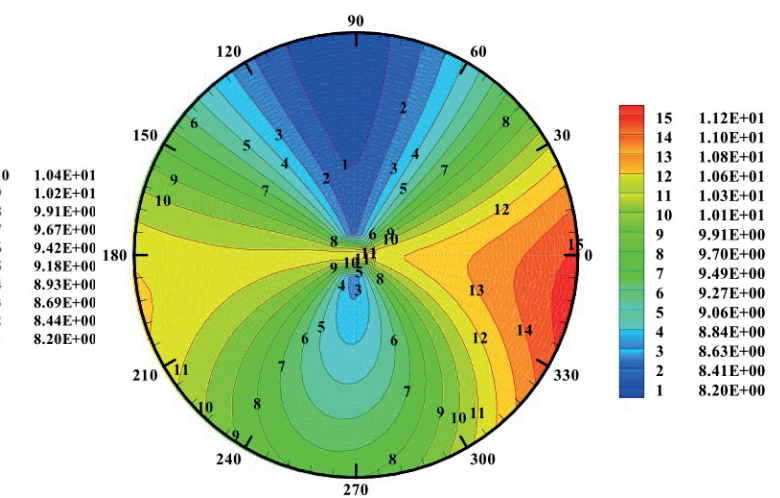

(b)

Fig. 18. Effect of no dimensional off-centering $(a b / R=0.2 ; b b / R=0.8)$ on dimensionless entropy generation of stagnation flow toward $a$ rotating disk with $R e=100$, in height of $\mathrm{Z}=1.4$ obtained by the Keller-Box method $\alpha=7$. 


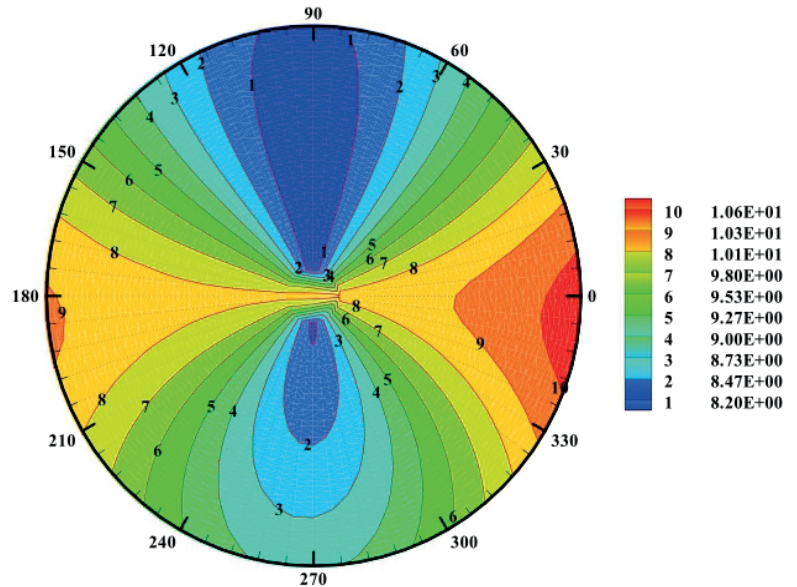

(a)

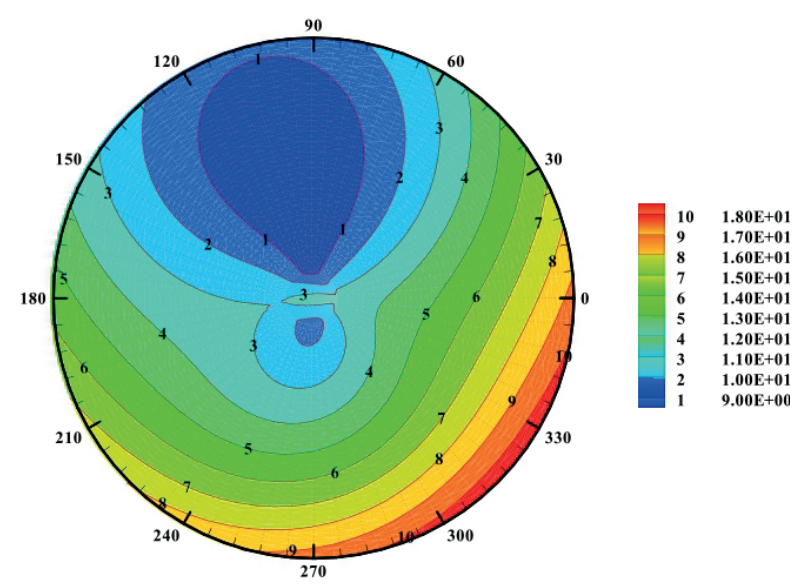

(b)

Fig. 19. Effect of Reynolds number (a $R e=10$; $\mathrm{b} R e=100$ ) on dimensionless entropy generation of stagnation flow toward a rotating disk, for no dimensional off-centering $b / R=0.4$, in height of $Z=1.4$ obtained by the Keller-Box method $\alpha=7$.

\section{Conclusions}

In this paper, off-centered stagnation flow towards a rotating disc has been solved by the Keller-Box method to find numerical solutions. And after finding velocity distributions, the mathematical formulation for the entropy generation analysis has been derived. The results of the present study, in a special case, were compared with those of previously published studies. Excellent agreement was obtained. The effect of physical parameters such as rotation ratio, off-centering, Reynolds number and height on axial direction are shown and discussed and physical results have been gotten. The present computations have provided some further insights into the thermodynamics of proposed rotating disk by entropy analysis, because the main goal of this research is to allow the designer to consider this important thermodynamics phenomenon in calculations of such systems.

\section{References}

[1] E. Jones, Journal of Computational Physics 40 (1981) 411-429.

[2] M. Zuki, Mathematical Models for the Boundary Layer Flow Due To A Moving Flat Plate, Universiti Teknologi Malaysia (2004).

[3] H.B. Keller, Numerical Methods in Boundary Layer Theory, Ann. Rev. Fluid Mech. 10 (1978) 417-433.

[4] H.B. Keller and T. Cebeci, Accurate Numerical Methods for Boundary Layer Flow-I, Two Dimensional Laminar Flows, In Proc. of the 2nd International Conference on Numerical Methods in Fluid Dynamics, Berkeley, California, 8 (1971) 92-100.

[5] H.B. Keller and T. Cebeci, Accurate Numerical Methods for Boundary Layer Flows-II, Two Dimensional Turbulent Flows, AIAA Journal 10 (9) (1972) 1193.

[6] G. Wilks and R. Hunt, Vertical Mixed Convection Row about a Horizontal Line Source of Heating or Cooling, International journal of Heat Mass Transfer 30 (6) (1987) 1119.

[7] G. Wilks and R. Hunt, The Assimilation of a Strong, TwoDimensional Laminar Jet into an Aligned Uniform Stream, the R. Sot. Edinburgh Proc. Sec. A Maths 90 (1) (1981) 13-23.

[8] G. Wilks, R. Hunt and D.S. Riley, The Two-Dimensional Laminar Vertical Jet with Positive or Adverse Buoyancy, Numerical Heat Transfer 8(4) (1985) 449-468.

[9] G. Wilks and R. Hunt, The Axisymmetric, Turbulent, Buoyant Jet Numerical Solution for a Semi Empirical Theory, Numerical Heat Transfer 9(4) (1986) 495-509.

[10] R. Hunt and G. Wilks, On the Behaviour of the Laminar Boundary Layer Equations of Mixed Convection Near a Point of Zero Skin Friction, Journal of Fluid Mech. 101 (2) (1980) 377-391.

[11] M.M. Rashidi, S. Abelman and N. Freidooni Mehr, Entropy Generation in Steady MHD Flow due to a Rotating Porous Disk in a Nanofluid, International Journal of Heat and Mass Transfer 62 (2013) 515-525.

[12] T.V. Kármán, Über laminare und turbulente Reibung, ZAMMJournal of Applied Mathematics and Mechanics/Zeitschrift für Angewandte Mathematik und Mechanik 1(4) (1921) 233-252.

[13] W.G. Cochran, The Flow due to a Rotating Disc, Proceedings of Cambridge Philosophical Society 30 (3) (1934) 365-375.

[14] J.T. Stuart, On the Effects of Uniform Suction on the Steady Flow due to a Rotating Disk, The Quarterly Journal of Mechanics and Applied Mathematics 7 (1954) 446-457.

[15] E.M. Sparrow and J.L. Gregg, Mass Transfer, Flow, and Heat Transfer about a Rotating Disk, ASME Journal of Heat Transfer 82 (4) (1960) 294-302.

[16] K. Hiemenz, Die Grenzschicht an Einem in den Gleichformingen Flussigkeitsstrom Eeingetauchten Graden Kreiszylinder. Dinglers Polytechnic Journal 326 (1911) 321-324.

[17] C.Y. Wang, The Three Dimensional Flow due to a Stretching Flat Surface, Phys. Fluids, 27 (1984) 1915-1917. 
[18] M. M. Rashidi, T. Hayat, E. Erfani, Simultaneous Effects of Partial Slip and Thermal-Diffusion and Diffusion-Thermo on Steady MHD Convective Flow due to a Rotating Disk, Communications In Nonlinear Science And Numerical Simulation 16 (11) (2011) 4303-4317.

[19] M. M. Rashidi and S. Dinarvand, Purely Analytic Approximate Solutions for Steady Three-Dimensional Problem of Condensation Film on Inclined Rotating Disk by Homotopy Analysis Method, Nonlinear Analysis Real World Applications 10 (4) (2009) 2346-2356.

[20] M.M. Rashidi and H. Shahmohamadi, Analytical Solution of Three-Dimensional Navier-Stokes Equations for the Flow near an Infinite Rotating Disk, Communications In Nonlinear Science And Numerical Simulation 14 (7) (2009) 2999-3006

[21] .M. Rashidi, S.A. Mohimanian Pour, Analytic Solution of Steady Three-Dimensional Problem of Condensation Film on Inclined Rotating Disk by Differential Transform Method, Mathematical Problems In Engineering Volume 2010 (2010), Article ID 613230, 15 pages doi:10.1155/2010/613230.

[22] S. Dinarvand, M.M. Rashidi and H. Shahmohamadi, Analytic Approximate Solution of Three-Dimensional Navier-Stokes Equations of Flow between Two Stretchable Disks, Numerical Methods for Partial Differential Equations 26 (6) (2010) 15941607.

[23] M.M. Rashidi, H. Shahmohamadi and G. Domairry, Variational Iteration Method for Solving Three-Dimensional NavierStokes Equations of Flow between Two Stretchable Disks, Numerical Methods for Partial Differential Equations 27(2) (2011) 292-301.

[24] S. Ad'boud, S. Saouli, Entropy analysis for viscoelastic magneto hydrodynamic flow over a stretching surface, Int. J. Non Linear Mech. 45 (5) (2010) 482-489.

[25] A.S. Butt, S. Munawar, A. Ali, A. Mehmood, Entropy generation in the Blasius flow under thermal radiation, Phys. Scr. 85 (3) (2012) 035008.

[26] J.Y. San, W.M. Worek, Z. Lavan, Entropy generation in combined heat and mass transfer, Int. J. Heat Mass Transfer 30 (7) (1987) 1359-1369.

[27] A. Bejan, in: P.H. James, F.I. Thomas (Eds.), Advances in Heat Transfer, 1982.

[28] C.Y. Wang, Off-centered stagnation flow towards a rotating disc, Int. J. Eng. Sci. 46 (2008) 391-396.

[29] C.G. Carrington and Z.F. Sun, Second law analysis of combined heat and mass transfer phenomena, Int. J. Heat Mass Transfer 34 (1991) 2767-2773.

[30] S. Mahmud and R.A. Fraser, The second-law analysis in fundamental heat transfer problems, Int. J. Therm. Sci. 42 (2003) 177-186.

[31] N. Bouzid, S. Saouli and S. Aiboud-Saouli, Entropy generation in ice slurry pipe flow, Int. J. Refrig. 31 (2008) 1453-1457.

[32] A. Arikoglu, I. Ozkol and G. Komurgoz, Effect of slip on entropy generation in a single rotating disk in MHD flow, Applied Energy 85 (12) (2008) 1225-1236.

[33] A. Bejan, Entropy generation minimization: the method of thermodynamic optimization of finite-size systems and finitetime processes, CRC Press, 1996.

[34] F. Homann, Der Einfluss grosser Zahigkeit bei der Stromung um den Zylinder und um die Kugel, ZAMM 16 (1936) 153-164.

[35] E. Erfani, M.M. Rashidi, A. Basiri parsa, The modified Differential Transform Method for solving off-centered stagnation flow towards a rotating disc, International Journal of Computational Methods 7(4) (2010) 655-670.

Received November 23, 2013; accepted December 26, 2013. 